02

\title{
Наблюдение когерентных осцилляций населенности NV-центров в алмазе в микроволновом диапазоне
}

\author{
(C) Р.А. Ахмеджанов ${ }^{1}$, Л.А. Гущин ${ }^{1}$, И.В. Зеленский ${ }^{1}$, В.А. Низов ${ }^{1,2}$, Н.А. Низов ${ }^{1,2}$, Д.А. Собгайда ${ }^{1}$ \\ ${ }^{1}$ Институт прикладной фризики РАН, \\ 603950 Нижний Новгород, Россия \\ ${ }^{2}$ ФИЦ Казанский научный центр РАН, \\ 420111 Казань, Россия \\ e-mail: rinat@appl.sci-nnov.ru
}

Поступила в редакцию 21.10.2020 г.

В окончательной редакции 04.12.2020 г.

Принята к публикации 04.12.2020 г.

Приведены результаты экспериментов по наблюдению особенностей в спектре оптически детектируемого магнитного резонанса в ансамблях NV-центров в алмазе при комнатной температуре при возбуждении низкочастотных переходов двухчастотным и амплитудно модулированным одночастотным микроволновым излучением. Исследована зависимость параметров этих особенностей от интенсивности оптического излучения и мощности микроволнового излучения.

Ключевые слова: NV-центры в алмазе, оптически детектируемый магнитный резонанс, релаксация населенности, когерентные осцилляции населенности, выжигание спектральных провалов.

DOI: $10.21883 /$ OS.2021.03.50656.265-20

\section{Введение}

NV-центры в алмазе часто используются в качестве рабочей среды для устройств зондирования магнитных и электрических полей [1,2], температуры [3], реализации квантовых вычислений [4], а также стабильных флуоресцентных биомаркеров [5]. Это связано с сочетанием удобства оптического детектирования квантового состояния и длинных времен жизни когерентности даже при комнатной температуре. Основное состояние NVцентров имеет спиновые подуровни, переходы между которыми лежат в микроволновом диапазоне. Благодаря оптической поляризации спина и спин-зависимой флуоресценции NV-центров широко распространено применение метода оптически детектируемого магнитного резонанса (ОДМР). Одним из направлений исследований в последнее время является использование комбинированного возбуждения NV-центров несколькими электромагнитными волнами. Например, наблюдение ОДМР при дополнительном приложении радиочастотного излучения [6-8]. С точки зрения приложений перспективным выглядит метод двухквантовой магнитометрии [9] (использующий когерентную манипуляцию состоянием $\mathrm{NV}$-центра, имеющим подуровни с проекцией спина \pm 1 , при помощи двухчастотного поля), позволяющий исключить влияние температуры/электрического поля и повысить чувствительность. В микроволновом диапазоне при комнатной температуре наблюдались эффекты когерентного пленения населенности [10,11], АутлераТаунса $[12,13]$. С точки зрения изучения динамики NVцентров предлагается наблюдение выжигания спектраль- ных провалов $[14,15]$ и когерентных осцилляций населенности [16,17].

Когерентные осцилляции населенности (КОН или CPO, Coherent Population Oscillations) - это явление, известное в области квантовой оптики. Они проявляются как узкий провал (резонанс КОН) в линии поглощения при сканировании частоты пробного излучения вблизи частоты одновременно приложенного к среде возбуждающего излучения с постоянной частотой. В отличие от обычного спектрального провала, ширина которого ограничена однородным уширением $1 / T_{2}$, ширина резонанса КОН может быть намного меньше и соответствует скорости релаксации населенности $1 / T_{1}$. В некоторых работах такую узкую особенность связывают с сильной дисперсией и возможностью наблюдения „медленного“ света (см., например, [18]) и реализации оптической памяти [19]. Существует другая точка зрения: наблюдаемые незначительные замедления световых импульсов можно объяснить искажением их формы при распространении через насыщающийся поглотитель, а узкий провал некорректно относить чисто к поглощению пробного излучения [20].

Название эффекта когерентных осцилляций населенности отражает объяснение наблюдения такого узкого спектрального провала. При одновременном приложении пробного излучения и накачки в суммарном возбуждающем излучении присутствуют биения. Если частота этих биений мала по сравнению со скоростью релаксации населенности, то населенность среды успевает отслеживать изменения в уровне эффективной накачки (считается, что интенсивность накачки и пробного излучения достаточно большая чтобы влиять 
на населенность). Таким образом, населенность среды осциллирует с частотой отстройки пробного излучения от накачки. Такой нестационарный отклик среды приводит к возникновению узкого провала при сканировании частоты пробного излучения вблизи частоты накачки с характерным частотным масштабом $1 / T_{1}$.

Настоящая работа посвящена экспериментальному наблюдению когерентных осцилляций населенности в ансамбле NV-центров в алмазе при использовании двухчастотной микроволновой накачки и амплитудной модуляции одночастотного СВЧ излучения.

\section{Эксперимент}

Эксперименты проводились при комнатной температуре. Оптическая часть установки собрана по конфокальной схеме. Образец - НРНТ (high-pressure-hightemperature)-алмаз производства Element Six, предоставленный ФИАН им. Лебедева, где он был облучен электронным пучком дозой $10^{18}$ electron $/ \mathrm{cm}^{2}$ и отожжен для повышения концентрации NV-центров. Для возбуждения $\mathrm{NV}$-центров использовалось излучение накачки на длине волны $532 \mathrm{~nm}$ (мощностью несколько десятков $\mathrm{mW}$ ), сфокусированное в пятно диаметром $100 \mu \mathrm{m}$. Флуоресценция детектировалась в диапазоне длин волн, большем $600 \mathrm{~nm}$, при помощи лавинного фотодиода. Образец помещался в магнитное поле, направленное вдоль направления [111], для выделения одной из 4групп NVцентров по направлению оси симметрии в кристаллической решетке алмаза. По спектру ОДМР определялась частота микроволнового перехода $0 \rightarrow+1$ для этой группы.

В первой серии экспериментов использовалась стандартная схема с двумя генераторами. Генераторы были синхронизованы по общему опорному сигналу для обеспечения когерентности используемых полей. Во избежание влияния генераторов друг на друга использовались вентили; после сложения суммарный сигнал усиливался и поступал на антенну, выполненную по схеме магнитной петли диаметром $8 \mathrm{~mm}$ (образец помещался в центр петли). На рис. 1, $a$ показан типичный спектр ОДМР. Частота одного из генераторов фиксировалась в центре пика ОДМР (управляющее поле), в то время как частота второго (пробное поле) сканировалась. Видно, что при возбуждении двумя генераторами происходят кардинальные изменения в форме ОДМР: во-первых, выжигается спектральный провал, во-вторых, на его фоне появляется узкий пик, который, как и в работе [16], можно аппроксимировать двумя контурами (в нашем случае их ширины $\sim 30$ и $\sim 1.5 \mathrm{kHz}$, рис. $1, b)$. При медленном сканировании частоты пробного излучения и усреднении по малому числу реализаций когерентные осцилляции населенности наблюдались напрямую, проявляясь в виде осцилляций сигнала флуоресценции во времени (рис. 1,c). Переход к проявлению КОН в виде
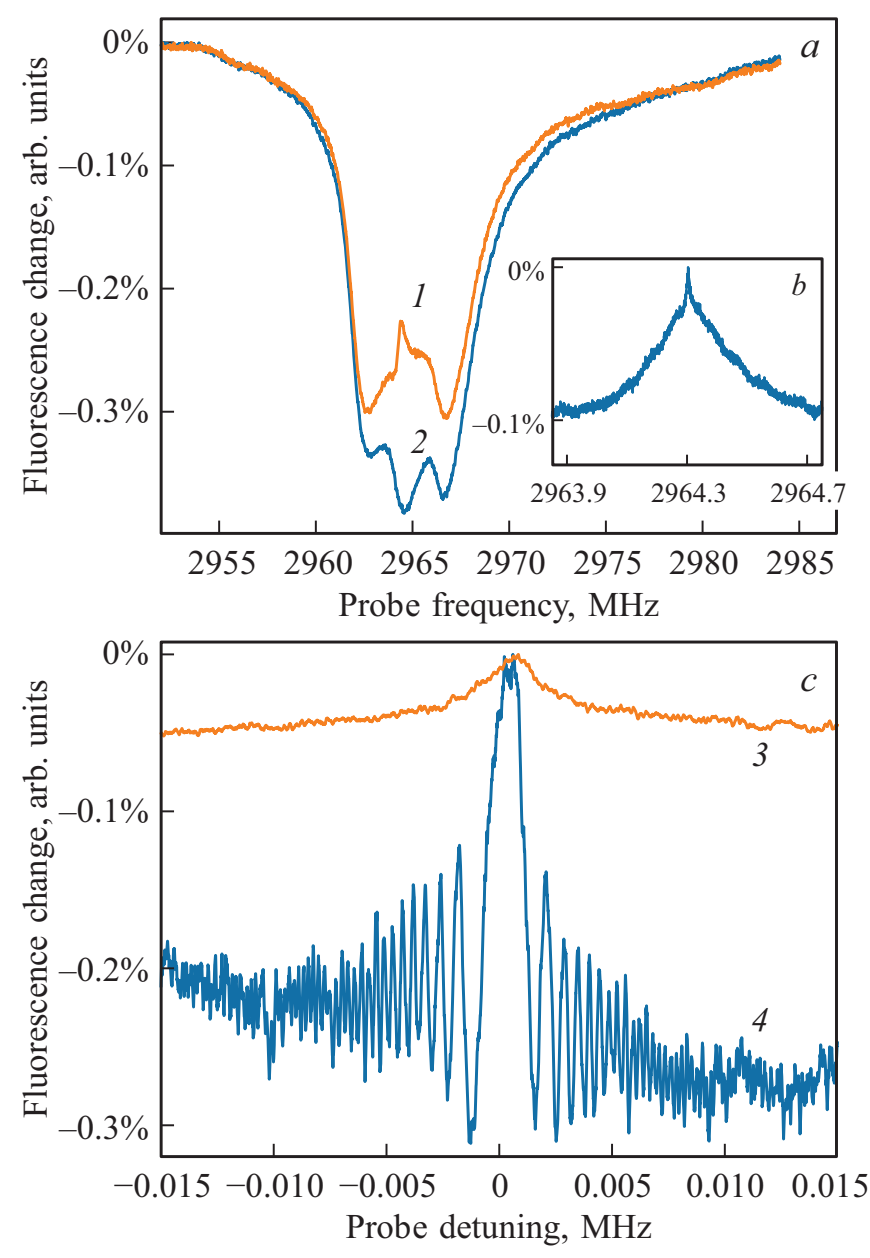

Рис. 1. Измеренное изменение интенсивности флуоресценции образца при сканировании частоты пробного поля $(a)$ : 1 - спектр ОДМР при приложении управляющего поля на частоте $2964.3 \mathrm{MHz}, 2$ - в отсутствии управляющего поля. На вставке $b$ показана зависимость флуоресценции от частоты пробного излучения в более узких пределах вблизи частоты управляющего поля. (c) Зависимость интенсивности флуоресценции от частотной отстройки пробного излучения в диапазоне $\pm 15 \mathrm{kHz}$. Длительность сканирования $\sim 16 \mathrm{~ms}$. Результат после усреднения по 700 реализациям (3) и 5 реализациям (4).

узкого спектрального провала (резонанса КОН) происходит при усреднении по большому числу реализаций (так как относительная фаза двух возбуждающий полей не сохранялась от реализации к реализации).

Заметим, что в основе когерентных осцилляций населенности лежат биения накачки и пробного излучения, и требуется взаимная когерентность генерируемых полей. Это накладывает ограничения на стабильность частоты генераторов и требует их синхронизации. Также для наблюдения КОН с наилучшим контрастом амплитуды двух полей должны совпадать.

Заметим, что требование когерентности полей и оптимальный контраст КОН автоматически реализуются при переходе от использования двух генераторов к глубокой 


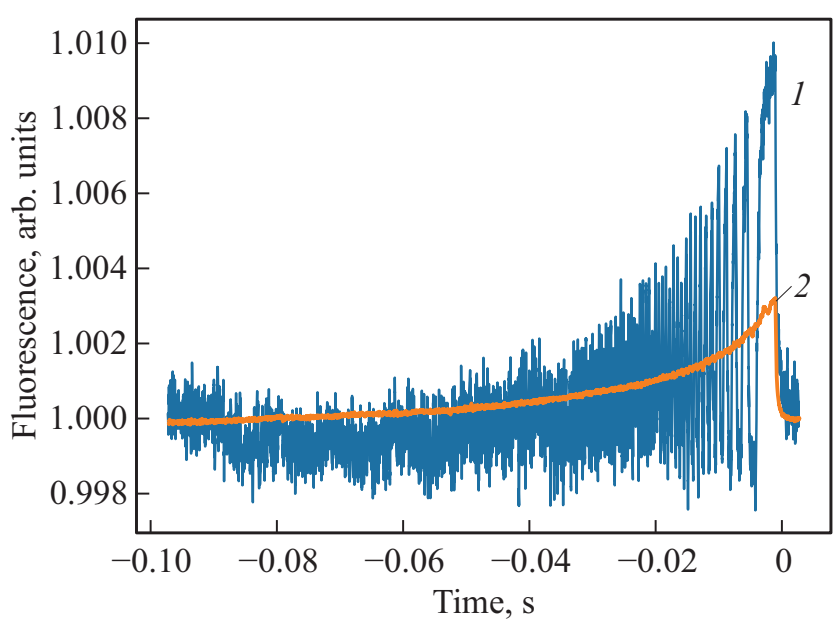

Рис. 2. Изменение интенсивности флуоресценции при линейном сканировании частоты модуляции за $0.1 \mathrm{~s}$ от $10 \mathrm{kHz}$ до $1 \mathrm{mHz}$ (при времени, большем 0, начинается следующее сканирование от $10 \mathrm{kHz}$ ). Показаны одиночная реализация (1) и результат усреднения по большому числу реализаций (2).

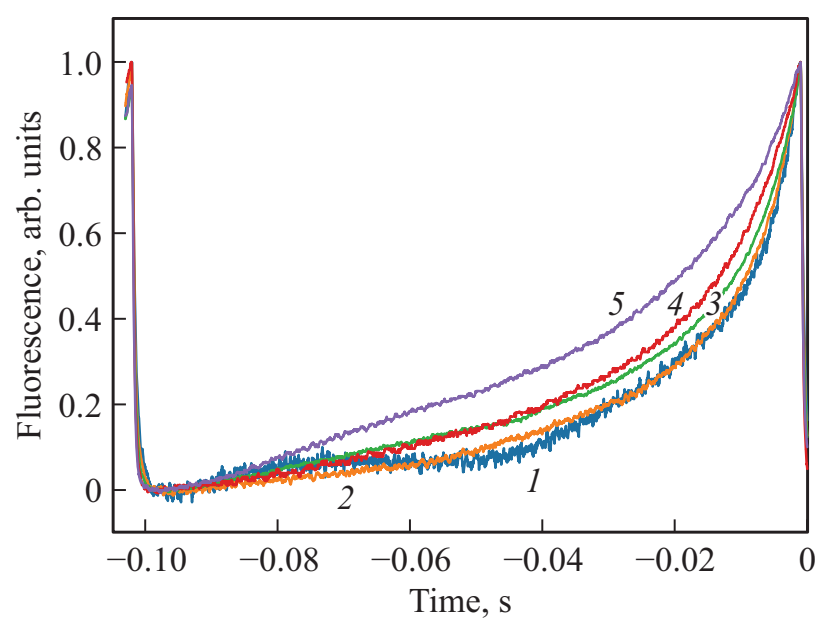

Рис. 3. Спад КОН при различной мощности оптической накачки: 1.2 (1), 3 (2), 5.9 (3), 12 (4) и $23 \mathrm{~mW}$ (5). Параметры сканирования частоты амплитудной модуляции те же, что на рис. 2 (скорость сканирования $100 \mathrm{kHz} / \mathrm{s}$ ). Сигнал флуоресценции нормирован на контраст.

(полной) амплитудной модуляции излучения одного генератора. В этой связи во второй серии экспериментов мы использовали амплитудно модулированное (меандром) микроволновое излучение одного генератора. Для этих целей в микроволновой части между генератором СВЧ излучения и усилителем присутствовал переключатель, при помощи которого создавалась амплитудная модуляция (включение/выключение). Модулированная накачка действует аналогично биениям между излучениями двух генераторов. Следует отметить, что в работе [16] рассмотрено отличие между амплитудной модуляцией (синусоидальной) и приложением двух полей. Отмечается, что для низкочастотной амплитудной модуляции также следует ожидать наблюдение когерентных осцилляций населенности. На рис. 2 показан характерный вид наблюдаемого сигнала флуоресценции образца при изменении во времени частоты амплитудной модуляции приложенного СВЧ излучения. В низкочастотной области заметны осцилляции в уровне сигнала, пропадающие при частотах модуляции больше $\sim 2 \mathrm{kHz}$. Осцилляции флуоресценции соответствует тому, что в процессе измерения включается/выключается микроволновая накачка. Если частота модуляции много меньше $1 / T_{1}$, то в сигнале флуоресценции фактически должны наблюдаться переключения между двумя уровнями, разница между которыми соответствует контрасту при наблюдении ОДМР. Если частота модуляции много больше $1 / T_{1}$, то на NV-центры эффективно воздействует СВЧ излучение с вдвое меньшей (усредненной) мощностью по сравнению с непрерывно включенной накачкой без модуляции, и наблюдается соответствующий такой мощности уровень флуоресценции.

При усреднении по большому числу отдельных реализаций (фаза амплитудной модуляции в каждой реализации не сохраняется) колебания в уровне флуоресценции исчезают и формируется пик с характерным частотным масштабом спада $\sim 1 \mathrm{kHz}$ (рис. 2). Этот спад проявление когерентных осцилляций населенности, аналогичный узкому провалу, наблюдаемому в схеме с использованием пробного излучения. Как величина, так и частотный масштаб этого спада характеризуют ансамбль NV-центров в образце как нелинейную среду c характерным временем (например, насыщающийся поглотитель). Величина спада соответствует разнице между уровнем флуоресценции при средней мощности СВЧ излучения (половина от полностью включенной) и средним значением между сигналом при включенной и выключенной накачке. Эта разница может наблюдаться в случае нелинейной зависимости уровня флу-

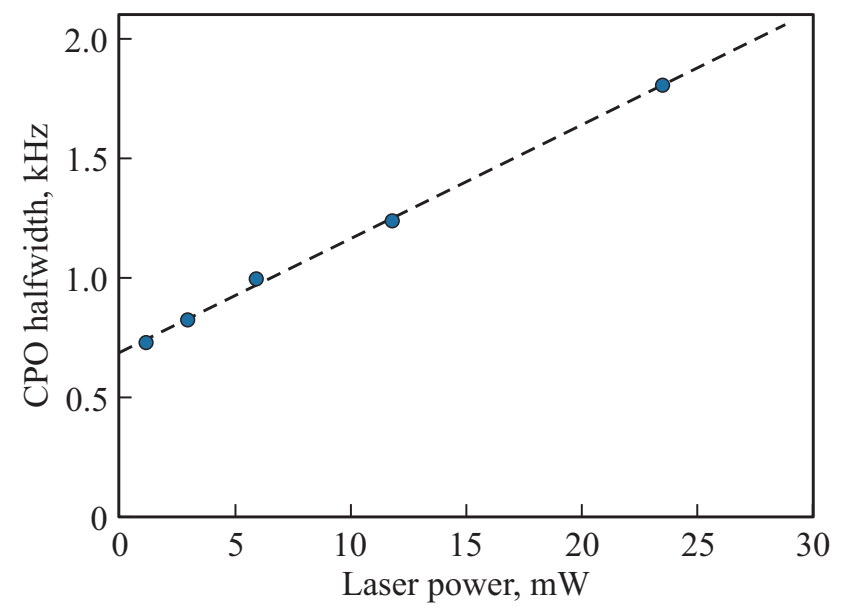

Рис. 4. Зависимость полуширины спада КОН от мощности возбуждающего оптического излучения. Кругами отмечены значения, полученные из рис. 3 по уровню 0.5. Штриховая линия - линейная аппроксимация. 
оресценции от мощности приложенного СВЧ излучения. В нашем случае нелинейность присутствует из-за насыщения ОДМР (глубина провала во флуоресценции при его наблюдении ограничена). Поэтому при сравнении половинной и полной мощности накачки контраст ОДМР (уменьшение флуоресценции) возрастает меньше чем в два раза. Этим объясняется относительное уменьшение средней флуоресценции при высокочастотной модуляции. Характерный частотный масштаб спада соответствует обратному времени жизни населенности $1 / T_{1}$.

\section{Возможности использования изменений в спектре ОДМР при одновременном возбуждении двух микроволновых полей}

Измерение параметров описанных выше изменений в спектре ОДМР кажется весьма перспективным методом для исследования релаксационных процессов в образцах алмаза, активированных NV-центрами. Действительно, измерение ширин спектральных провалов и резонансов КОН может дать информацию о характерных значениях времен поперечной и продольной релаксаций. Однако, как справедливо отмечено в работе [16], следует помнить, что измеренные константы релаксации характеризуют систему, возмущенную светом и СВЧ излучением, так что сравнение с другими измерениями требует экстраполяции измеренных результатов на нулевую интенсивность света и мощность микроволнового излучения. Мы провели несколько экспериментов для проверки влияния характеристик микроволнового и оптического полей на величины измеряемых констант.

Влияние интенсивности оптической накачки

Были проведены наблюдения спада КОН при различных уровнях оптического возбуждения. С увеличением интенсивности оптической накачки заметно увеличение характерного частотного масштаба резонанса КОН (рис. 3 и 4). Это объясняется тем, что с увеличением интенсивности растет скорость оптической поляризации электронного спина NV-центров, что играет роль дополнительного канала релаксации, увеличивая как равновесную населенность подуровня 0, так и скорость релаксации к новому равновесию при возмущении (например, при выключении СВЧ излучения). Экстраполяция к нулевому значению интенсивности (рис. 4) приводит к характерным скоростям продольной релаксации порядка $500 \mathrm{~Hz}$ при использовании амплитудной модуляции микроволнового излучения одного генератора, что соответствует измерениям так называемым методом relaxation in the dark (релаксация в „темно$\mathrm{Te}^{\text {“6 })}[21,22]$. При этом для возбуждения NV-центров использовалась оптическая импульсно-периодическая последовательность с варьируемой частотой следования импульсов. По переднему фронту импульса флуоресценции определялась степень поляризации NV-центра, ее
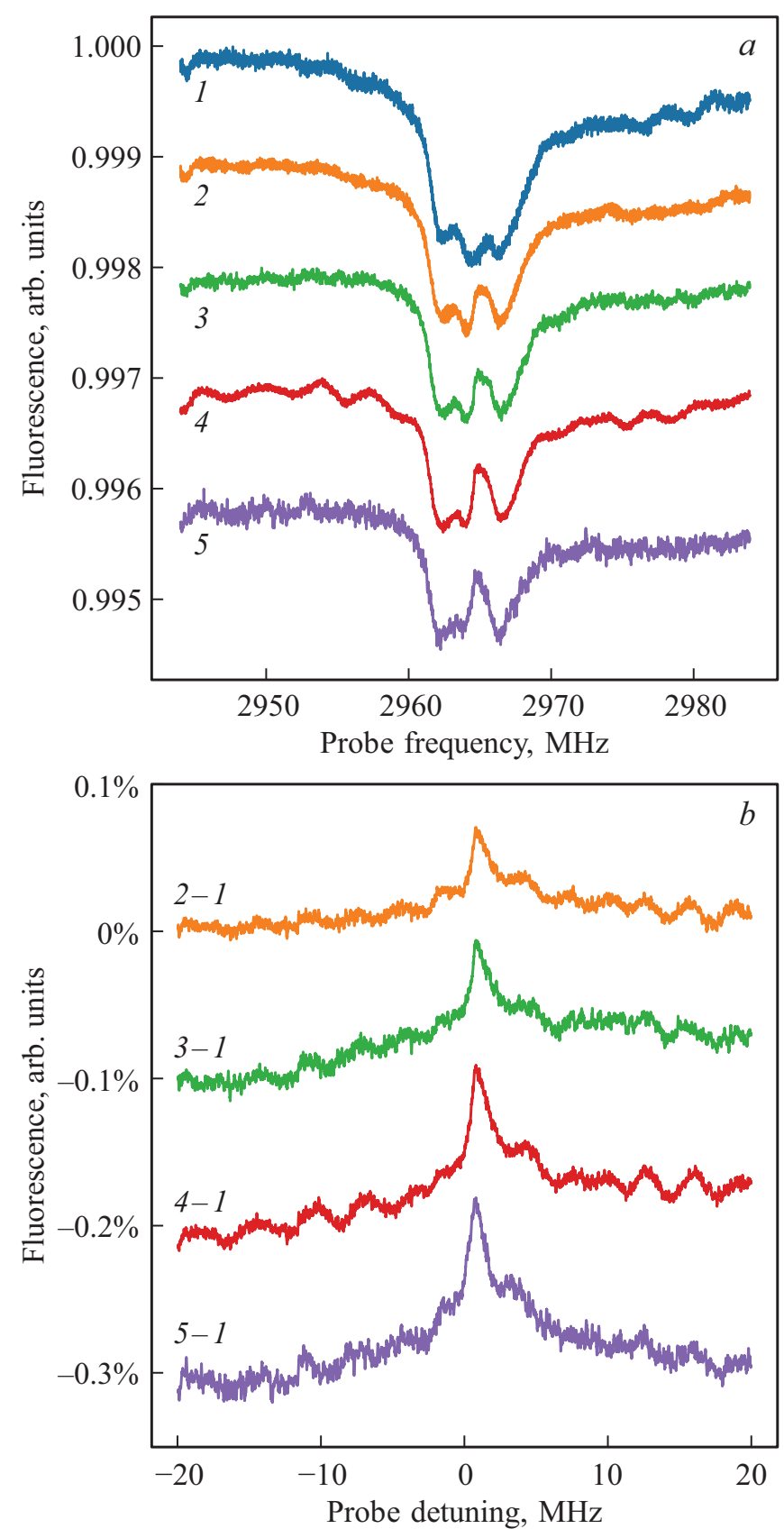

Рис. 5. Выжигание спектрального провала в линии ОДМР при приложении управляющего поля на частоте $2964.64 \mathrm{MHz}$ с различной мощностью: без управляющего поля (1), относительная мощность $-9(2),-6(3 \mid /),-3(4)$ и $0 \mathrm{~dB}(5)$. Приведены нормированный сигнал флуоресценции $(a)$ и изменение относительно сигнала без управляющего поля $(b$, из каждой кривой вычитается кривая 1). Кривые смещены по вертикали для наглядности.

зависимость от периода следования позволяла определить время деполяризации, т. е. релаксации населенности нижних уровней.

Влияние мощности микроволновой накачки

В экспериментах также исследовалось влияние мощности используемого микроволнового излучения на ха- 


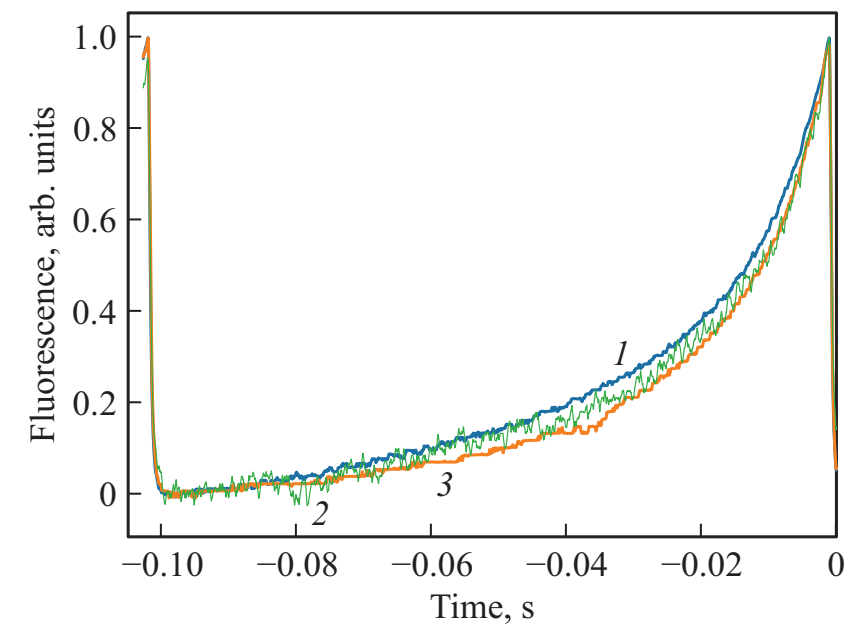

Pис. 6. Спад КОН при различной мощности СВЧ излучения: относительные мощности 0 (1), -5 (2) и $-10 \mathrm{~dB}$ (3). Параметры сканирования частоты амплитудной модуляции те же, что на рис. 2 (скорость сканирования $100 \mathrm{kHz} / \mathrm{s}$ ). Сигнал флуоресценции нормирован на контраст.

рактерные значения ширин выжигаемых спектральных провалов и резонансов КОН. Как видно из рис. 5, увеличение мощности управляющего поля приводит лишь к увеличению глубины спектрального провала, практически не изменяя его ширину (в диапазоне используемых нами мощностей до нескольких ватт). Это значит, что в этом диапазоне мощность СВЧ излучения достаточно мала и не приводит к полевому уширению. При этом характерное значение ширины провала составляет порядка $2 \mathrm{MHz}$. Характерные ширины спадов КОН в наших условиях также практически не чувствительны к мощности микроволнового излучения (рис. 6).

\section{Заключение}

В настоящей работе представлены результаты экспериментов по наблюдению когерентных осцилляций населенности NV-центров в алмазе в микроволновом диапазоне при комнатной температуре. Использовались различные варианты накачки: двухчастотное микроволновое излучение и амплитудно модулированное одночастотное СВЧ излучение. Исследована зависимость параметров резонансов КОН и выжигаемых спектральных провалов от интенсивности оптического излучения и мощности микроволнового излучения. Показано, что диагностика динамики релаксации в ансамблях NV-центров с использованием резонансов КОН и выжигаемых спектральных провалов возможна лишь с учетом влияния оптического и микроволнового излучения на релаксационные процессы.

\section{Финансирование работы}

Работа выполнена в рамках государственного задания Министерства науки и высшего образования Российской Федерации (тема № АААА-А19-119011790156-3).

\section{Конфликт интересов}

Авторы заявляют, что у них нет конфликта интересов.

\section{Список литературы}

[1] Taylor J.M., Cappellaro P., Childress L., Jiang L., Budker D., Hemmer P.R., Yacoby A., Walsworth R., Lukin M.D. // Nature Physics. 2008. V. 4. P. 810. doi 10.1038/nphys 1075

[2] Akhmedzhanov R., Gushchin L., Nizov N., Nizov V., Sobgayda D., Zelensky I., Hemmer P. // Phys. Rev. A. 2017. V. 96. N 1. P. 013806. doi 10.1103/PhysRevA.96.013806

[3] Kucsko G., Maurer P.C., Yao N.Y., Kubo M., Noh H.J., Lo P.K., Park H., Lukin M.D. // Nature. 2013. V. 500. P. 54. doi 10.1038 /nature12373

[4] Gurudev Dutt M.V., Childress L., Jiang L., Togan E., Maze J., Jelezko F., Zibrov A.S., Hemmer P.R., Lukin M.D. // Science. 2007. V. 316. P. 1312. doi 10.1126/science. 1139831

[5] Alkahtani M.H., Alghannam F., Jiang L., Almethen A., Rampersaud A.A., Brick R., Gomes C.L., Scully M.O., Hemmer P.R. // Nanophotonics. 2018. V. 7. P. 1423-1453. doi 10.1515/nanoph-2018-0025

[6] Dmitriev A.K., Chen H.Y., Fuchs G.D., Vershovskii A.K. // Phys. Rev. A. 2019. V. 100. N 1. P. 011801. doi 10.1103/PhysRevA.100.011801

[7] Tashima T., Morishita H., Mizuochi N. // Phys. Rev. A. 2019. V. 100. N 2. P. 023801. doi 10.1103/PhysRevA.100.023801

[8] Rohr S., Dupont-Ferrier E., Pigeau B., Verlot P., Jacques V., Arcizet O. // Phys. Rev. Lett. 2014. V. 112. N 1. P. 010502. doi 10.1103/PhysRevLett.112.010502

[9] Mamin H.J., Sherwood M.H., Kim M., Rettner C.T., Ohno K., Awschalom D.D., Rugar D. // Phys. Rev. Lett. 2014. V. 113. N 3. P. 030803. doi 10.1103/PhysRevLett.113.030803

[10] Jamonneau P., Hétet G., Dréau A., Roch J.F., Jacques V. // Phys. Rev. Lett. 2016. V. 116. N 4. P. 043603. doi 10.1103/PhysRevLett.116.043603

[11] Huillery P., Leibold J., Delord T., Nicolas L., Achard J., Tallaire A., Hétet G. // arXiv preprint. 2020. arXiv 2005.13082

[12] Morishita H., Tashima T., Mima D., Kato H., Makino T., Yamasaki S., Fujiwara M., Mizuochi N. // Scientific Reports. 2019. V. 9. N 1. P. 1. doi 10.1038/s41598-019-49683-Z

[13] Dong Y., Zheng Y., Chen X.D., Guo G.C., Sun F.W. // arXiv preprint. 2017. arXiv 1712.04582

[14] Kehayias P., Mrózek M., Acosta V.M., Jarmola A., Rudnicki D.S., Folman R., Gawlik W., Budker D. // Phys. Rev. B. 2014. V. 89. N 24. P. 245202. doi 10.1103/PhysRevB.89.245202

[15] Rosenzweig Y., Schlussel Y., Folman R. // Phys. Rev. B. 2018. V. 98. N 1. P. 014112. doi 10.1103/PhysRevB.98.014112

[16] Mrózek M., Wojciechowski A.M., Rudnicki D.S., Zachorowski J., Kehayias P., Budker D., Gawlik W. // Phys. Rev. B. 2016. V. 94. N 3. P. 035204. doi 10.1103/PhysRevB.94.035204

[17] El-Ella H.A.R., Huck A., Andersen U.L. // Phys. Rev. B. 2019. V. 100. N 21. P. 214407. doi 10.1103/PhysRevB.100.214407 
[18] Bigelow M.S., Lepeshkin N.N., Boyd R.W. // Phys. Rev. Lett. 2003. V. 90. N 11. P. 113903. doi 10.1103/PhysRevLett.90.113903

[19] Maynard M.A., Bretenaker F., Goldfarb F. // Phys. Rev. A. 2014. V. 90. N 6. P. 061801. doi 10.1103/PhysRevA.90.061801

[20] Zapasskii V.S., Kozlov G.G. // Opt. Spectrosc. 2006. V. 100. N 3. P. 419-424. doi 10.1134/S0030400X06030192

[21] Jarmola A., Acosta V.M., Jensen K., Chemerisov S., Budker D. // Phys. Rev. Lett. 2012. V. 108. N 19. P. 197601. doi 10.1103/PhysRevLett.108.197601

[22] Mrózek M., Rudnicki D., Kehayias P., Jarmola A., Budker D., Gawlik W. // EPJ Quantum Technology. 2015. V. 2. N 1. P. 1. doi 10.1140/epjqt/s40507-015-0035-z 